\title{
Análise de indicadores de produção científica e geração de conhecimento nas universidades estaduais paranaenses
}

\author{
Carlos Eduardo Caldarelli" \\ Claudia Perdigão** \\ Marcia Regina Gabardo da Câmara**** \\ João Paulo Alves dos Santos.**** \\ Solange de Cássia Inforzato de Souza ${ }^{* * * * * *}$
}

\section{Resumo}

O objetivo deste estudo é analisar a contribuição das universidades estaduais para a produção científica e a oferta de ensino superior no estado do Paraná. Utilizaram-se diversas fontes de dados, dentre as quais, dados das próprias instituições; da base de dados do CNPq; Capes e do Censo do Ensino Superior, realizado pela Secretaria de Ciência e Tecnologia do Paraná (SETI). Os resultados apontam as universidades estaduais paranaenses como os principais atores na geração de produção científica, na oferta de ensino superior, bem como destacam que essas instituições concentram a maioria dos recursos humanos de alta qualificação no estado, em termos de ensino e pesquisa acadêmica. Também, pode-se concluir com este estudo que há diferenças entre as instituições estudadas, tanto na oferta de vagas quanto na produção científica a partir dos indicadores analisados, com destaque para os indicadores da UEL, UEM e Unioeste.

Palavras-chave: Universidades. Produção científica. Ensino superior.

Professor Adjunto do Departamento de Economia da Universidade Estadual de Londrina. E-mail: carlos.caldarelli@gmail.com

** Graduanda em Economia pela Universidade Estadual de Londrina - Bolsista IC Fundação Araucária. E-mail: claudiaperdigao92@gmail.com

*** Professora Associada do Departamento de Economia da Universidade Estadual de Londrina. E-mail:mgabardo@sercomtel.com.br

**** Graduando em Economia pela Universidade Estadual de Londrina - Bolsista IC/CNPq. E-mail: pe_ibg@hotmail.com

Professora Associada do Departamento de Economia da Universidade Estadual de Londrina. E-mail: soinfor@uel.br

http://dx.doi.org/10.5335/rtee.v20i43.4595

SUBMISSÃO: 16/10/2013. ACEITE: 04/08/2014. 


\section{Introdução}

Estudos recentes apontam um importante papel das Instituições de Ensino Superior (IES) no desenvolvimento das regiões brasileiras. As IES tem um papel pivotal no processo de criação e disseminação de novos conhecimentos e inovações. Nesse contexto, destaca-se que as IES como agentes locais são tão importantes quanto o ambiente para o desenvolvimento de uma região. $\mathrm{O}$ grande desafio tem sido integrar as universidades ao contexto regional, logo o primeiro passo é o mapeamento da atuação dessas instituições (CHIARINI; VIEIRA; ZORZIN, 2012).

Como destacam Alvares, Kannebley Júnior e Carolo (2013), o propósito da universidade é combinar suas funções de ensino e de pesquisa para o desenvolvimento, ou seja, formação de pessoal qualificado a ser contratado pelo setor produtivo e a pesquisa científica, que se entende como informação científico-tecnológica. Como tais instituições estão inseridas em um contexto regional, são também importantes agentes no desenvolvimento regional, desde que as aludidas instituições compreendam e estejam alinhadas às demandas regionais, no que tange à formação de capital humano e que seu desenvolvimento científico e tecnológico responda às demandas locais e regionais.

O relatório da OCDE (2007) aponta que as IES contribuem para a capacidade de gerar e absorver inovações e desempenham um papel fundamental no desenvolvimento e na competitividade de um país, uma vez que a gestação de novas tecnologias se encontra atrelada à investigação científica e à produção do conhecimento. Nesse contexto, as universidades e os institutos de pesquisa configuram-se, cada vez mais, como elementos centrais no processo inovativo em uma relação cada vez mais intensa com as empresas.

No Brasil, a despeito do crescimento da produção científica nas regiões Norte-Nordeste na década de 2000, fruto da implementação de políticas federais, a região Sul-Sudeste concentra a produção bibliográfica, particularmente nos estados de São Paulo, Rio de Janeiro e Minas Gerais. Os estados da região Sul ocupam posição de destaque no ranking nacional: Rio Grande do Sul, Paraná e Santa Catarina representam 10,5\%, 7,38\% e 3,81\% da produção brasileira, respectivamente (CHIARINI; VIEIRA; ZORZIN, 2012).

Nesse contexto, o objetivo deste estudo é analisar a contribuição das universidades estaduais paranaenses para a produção científica e de ativos intelectuais e sua dinâmica no estado e na região Sul do Brasil. Para isso, selecionaram-se os indicadores de oferta de ensino universitário estadual, alocação de recursos para pesquisa, indicadores de produção de pesquisa científica, e indicadores de patentes, a 
partir da base de dados do CNPq, Capes e do Censo do Ensino Superior (SETI-PR), e do Inepe, bem como a das próprias instituições.

\section{Universidades, desenvolvimento científico, econômico e regional}

O presente capítulo está dividido em duas seções. A primeira seção discute a relação da universidade com a formação de capital humano e a segunda analisa 0 papel e a contribuição das universidades, em particular das universidades públicas, para o desenvolvimento regional.

\subsection{Instituições de ensino superior e qualificação de capital humano}

A educação e a sua contribuição para o crescimento e o desenvolvimento das nações têm sido alvo da pesquisa econômica desde os anos 1960. Nesse aspecto, destaca-se a teoria do crescimento endógeno, cujas bases repousam no fato de que a qualificação do trabalho contribui para o incremento da produtividade. Mincer (1958), Schultz (1960) e Becker (1962) preconizam que a maior instrução, a maior escolaridade e o crescente treinamento contribuem para melhores salários e para a melhoria do bem-estar dos agentes e da sociedade. Barbosa Filho e Pessoa (2010) discutem as evidências teóricas e empíricas dos efeitos da educação no crescimento e destacam que a melhoria da qualidade do trabalho enquanto fator fundamental que contribui para maior produtividade do trabalho é derivado do investimento em capital humano (educação e treinamento); dos efeitos da política econômica de retornos da educação; das regularidades do mercado de trabalho e da sua associação com a decisão de estudar - pessoas mais habilidosas investem mais em estudo e obtêm maiores retornos e/ou rendimentos. Por fim, o investimento em educação permite compreender as origens das desigualdades de renda.

Os estudos empíricos resenhados por Barbosa Filho e Pessoa (2010) encontram resultados que favorecem a teoria do capital humano. No entanto, os autores destacam que a maior escolaridade nem sempre causa incremento da renda, pois essa pode estar associada a habilidades inatas, que tornam menos custoso às pessoas habilidosas obter maior escolaridade e maiores salários, envolvendo mecanismos. ${ }^{1}$ Os autores também analisam os retornos privados e sociais da educação e sua relação com o crescimento da renda per capita, enquanto facilitador 
do progresso técnico e destacam a variação e o nível do capital humano. Nelson e Phelps (1966) partem do suposto que trabalhadores mais escolarizados são mais capazes de adotar e têm maior facilidade para utilizar novas tecnologias, enquanto Romer $(1989 ; 2001)$ afirma que o capital humano é importante para o desenvolvimento das novas ideias que promovem o desenvolvimento científico, tecnológico e o desenvolvimento econômico endógeno.

Neste contexto, a universidade é uma instituição que desempenha um papel fundamental na formação de mão de obra e de qualificação do capital humano A literatura econômica também destaca a importância das Instituições de Ensino Superior (IES) na qualificação formal do trabalho, no treinamento, na formação de mão de obra qualificada e no desenvolvimento de novas habilidades e novas ideias que contribuem para a inovação, o crescimento da produtividade, o crescimento e o desenvolvimento econômico (ALVARES; KANNEBLEY JÚNIOR; CAROLO, 2003).

A interação mais intensa entre as IES e o desenvolvimento regional, principalmente pós 1980, assim como o papel dessas instituições no desenvolvimento local modelou inclusive um novo contrato social entre as universidades e a sociedade. Observa-se que o financiamento dessas instituições está cada vez mais condicionado à sua contribuição direta para a economia (RAPINI, 2007).

Acemoglu, Johnson e Robinson (2001, 2002, 2005) destacam que a presença de instituições fortalecidas estimula o desenvolvimento econômico ao favorecer a acumulação de fatores, a inovação e a alocação de fatores de forma eficiente, enquanto sociedades com instituições fracas apresentam pior desempenho.

Para Rapini (2007), a contribuição das universidades para o processo de inovação nas firmas e o crescimento econômico ocorre, principalmente, via fonte de conhecimento científico para as atividades de pesquisa básica e de conhecimento especializado para a área tecnológica da firma. Essas instituições também contribuem mediante a formação de cientistas e engenheiros qualificados para atividades relacionadas à solução de problemas que emergem no processo inovador das firmas.

Segundo Goddard et al. (2003) e Goodard (1999), as universidades e as instituições estão inseridas no contexto regional, as quais são importantes agentes de promoção do desenvolvimento regional, e quando estão alinhadas às demandas regionais criam riqueza e promovem o crescimento econômico.

Souza (2008) observa que os retornos do investimento em capital humano, para o Brasil em 2004, apresentam uma correlação positiva com salários, educação formal e experiência profissional. Kroth e Dias (2012) mensuraram a contribuição dos capitais físico e humano sobre o produto per capita dos municípios da região 
Sul para o período de 1994 a 2004, verificando que o capital humano exerceu a maior influência no produto per capita. Ainda nessa linha, Nakabashi e Felipe (2007) verificaram a importância do capital humano no crescimento dos municípios paranaenses. Zaist, Nakabashi e Salvato (2010) pontuaram que o ensino superior apresentou o maior retorno para a renda e para o crescimento econômico no estado do Paraná em 2005. Silva (2013) estudou os efeitos da educação sobre o diferencial de salários dos mercados de trabalho formal e informal, no estado do Paraná em 2009 , tendo encontrado efeitos positivos.

\subsection{A integração das universidades e dos sistemas econômicos}

As universidades podem ser definidas como organizações de ensino e pesquisa responsáveis por gerar, sistematizar e difundir conhecimentos científicos e culturais (LOPES, 2003). Ainda segundo Lopes:

A universidade pública é um importante instrumento de transformação social e econômica em todos os países. Esta importância é ainda maior em países em desenvolvimento como o Brasil. Isto porque estas instituições são responsáveis por expressivas parcelas da geração e transmissão de conhecimentos, da produção, apoio e divulgação de pesquisas, da reprodução da inteligência nacional, da preservação e difusão de nossa cultura e da prestação de serviços à Nação (2010, p. 11).

As universidades, além de contribuir para o desenvolvimento do país, podem ter um impacto significativo sobre as regiões nas quais estão inseridas, sejam essas o estado ou a sua macrorregião. Embora tenha uma longa tradição na literatura internacional, grande parte dessa oriunda de estudos realizados pela OCDE, são poucos os trabalhos que exploram essa temática no Brasil.

Pesquisas anteriores discutiram os efeitos do modelo de universidade de pesquisa e seus efeitos na economia regional e no seu desenvolvimento, dentre os quais destacam-se Acs, Anselin e Varga (2002); Acs, Audretsch e Feldman (1994); e Anselin, Varga e Acs (2000). Ainda nesta linha, estudo de Silva e McComb (2012) analisa se há spillovers de conhecimento presentes no ambiente universitário, e se ele contribui para a existência de taxas mais elevadas de geração e sobrevivência de start-ups em áreas que são geograficamente próximas à universidade.

Florax (1992) e Florax e Folmer (1992), por sua vez, analisam o impacto do conhecimento das universidades na promoção da inovação e no desenvolvimento das empresas e das indústrias e encontram externalidades positivas, comprovando a promoção para o desenvolvimento regional. 
A experiência da integração das universidades com o sistema econômico foi largamente incentivada, segundo Soares (2002), nos países como os Estados Unidos, Coréia, Canadá, França e Japão. No caso do Brasil, os mecanismos de interação mais complexos entre esses dois setores começaram a desenvolver-se a partir da década de 1990, na qual a universidade fica responsável pela geração do conhecimento e a empresa apropria-se desse conhecimento, traduzindo-o em inovação.

Essa relação pode manifestar-se por meio das incubadoras de empresas, geralmente presentes nas instituições de ensino, da exploração de economias de aglomeração, dando origem aos clusters, que geralmente localizam-se próximo a regiões que dispõem de uma infraestrutura científico-tecnológica consolidada em universidades e institutos de pesquisa, como os casos de Campinas e São Carlos, por intermédio da Unicamp e da UFSCAR, respectivamente (VILLELA, 2009).

Os setores nos quais as universidades têm se destacado, cuja relação entre ciência e tecnologia é relativamente significativa, são: as engenharias, a biotecnologia, as tecnologias a laser e microeletrônica. Rapini (2007) também destaca os setores industriais, nos quais há mais interações, tais como, química, petroquímica, farmacêutica, semicondutores, computadores, instrumentos eletrônicos, equipamentos elétricos e aeroespaciais; e as áreas científicas de maior aplicabilidade setorial: ciência da computação, ciência dos materiais, química, informática, metalurgia, física e matemática.

De acordo com Lopes (2010), uma universidade gera externalidades positivas (spillovers) capazes de alterar a estrutura socioeconômica da região. Além da contribuição para mudanças incrementais nas instituições locais e do aumento no estoque de capital humano, a economia regional é beneficiada pelos gastos relacionados ao funcionamento dessas instituições. Esse benefício materializa-se com a dinamização da economia regional, com a geração de emprego, de renda e, consequentemente, de receita tributária para estados e municípios.

No que diz respeito às universidades públicas, o aludido autor afirma que essas geram, sistematizam e difundem conhecimentos científicos e culturais (LOPES, 2003). Para o autor:

A universidade pública é um importante instrumento de transformação social e econômica em todos os países. Esta importância é ainda maior em países em desenvolvimento como o Brasil. Isto porque estas instituições são responsáveis por expressivas parcelas da geração e transmissão de conhecimentos, da produção, apoio e divulgação de pesquisas, da reprodução da inteligência nacional, da preservação e difusão de nossa cultura e da prestação de serviços à Nação (2010, p. 11). 
Deve-se deixar claro, no entanto, que a simples presença de uma universidade pública numa determinada região não é garantia de seu desenvolvimento. São necessárias ações que possibilitem a integração da universidade com outros agentes responsáveis pelo desenvolvimento regional, dentre os quais, se destacam as empresas.

\section{Procedimentos metodológicos}

Os dados para mensurar o papel das universidades públicas estaduais paranaenses no desenvolvimento científico e tecnológico estadual foram extraídos das bases de dados da Secretaria de Estado de Ciência e Tecnologia (SETI), da Plataforma Lattes do Conselho Nacional de desenvolvimento Científico e Tecnológico (CNPq), do Censo do Ensino Superior (Inep) e do sistema GeoCapes, assim como por meio de informações coletadas nas próprias instituições de ensino. As informações extraídas da base de dados do SETI permitem visualizar o quadro das universidades estaduais paranaenses. Os dados obtidos a partir da Plataforma Lattes possibilitam identificar as transformações nos indicadores de C\&T, tal como os grupos de pesquisa distribuídos pelo Brasil e produção científica. As informações do Censo do Ensino Superior permitiram traçar a evolução das instituições paranaenses frente às demais instituições (públicas e privadas) no estado, assim como comparar à evolução da região Sul. Os dados do sistema GeoCapes dão suporte a análise referente aos cursos de pós-graduação.

O tratamento dos dados compreende o cálculo de estatísticas descritivas e cálculos elementares, tais como média, proporção e obtenção de taxas de crescimento em porcentagem, de maneira a avaliar a evolução desses.

\section{Resultados e discussão}

\section{$4.1 \mathrm{O}$ retrato das universidades estaduais paranaenses}

No estado do Paraná estão instaladas 185 Instituições de Ensino Superior (IES), sendo 164 privadas e 21 públicas. As instituições privadas dedicam-se basicamente ao ensino, por meio da oferta de cursos de graduação e especialização, destinando, desse modo, poucos recursos ao conhecimento científico, embora o número de grupos de pesquisa cadastrado no Diretório de Grupos de Pesquisa no Brasil do CNPq tenha crescido consideravelmente em um período de dez anos (2000 a 2010) ${ }^{2}$

Teoria e Evidência Econômica - Ano 20, n. 43, p. 313-336, jul./dez. 2014 
para as IES privadas. Neste sentido, as grandes responsáveis pela produção de conhecimento científico são as instituições públicas que se subdividem em instituições federais, estaduais e municipais.

O estado do Paraná tem o segundo maior número de IES estaduais públicas do país, cujo ranking é liderado pelo estado de São Paulo (53 campi de instituições públicas), sendo 13 estaduais contra quatro federais e quatro municipais. Ao considerar a região Sul do Brasil, a qual tem 15 instituições estaduais, a relevância do estado do Paraná é afirmada. Em 2011, das oito universidades estaduais que estavam instaladas na região Sul, seis localizavam-se no Paraná (INEP, 2011).

A capacidade de produção de conhecimento científico pode ser avaliada pela qualidade dos recursos humanos presentes em cada grupo (Tabela 1). Em 2011, dos 28.569 docentes em atividade no estado, tem-se que 12.268 atuavam em IES públicas, contra 16.301 docentes nas privadas, o que oferece uma média de 584 docentes por instituição pública e 99 docentes por instituição privada, destacando o fato do tamanho das IES privadas, em média, ser notadamente menor. Além disso, enquanto a média de mestres e doutores é de 222 e 273,7 , respectivamente, para as públicas, cada instituição privada contava com uma média de 45,5 mestres e 12,9 doutores. Tendo em vista o diferencial dos recursos humanos, o ambiente proporcionado pelas instituições públicas, nas quais profissionais altamente qualificados convivem e trabalham juntos, favorece a produção de conhecimento.

Tabela 1 - Total de docentes em exercício, total de instituições e média de mestres e doutores por instituição por categoria administrativa das IES paranaenses - 2011

\begin{tabular}{|c|c|c|c|c|c|c|}
\hline \multirow{2}{*}{$\begin{array}{l}\text { Unidade da Federação / } \\
\text { categoria administrativa }\end{array}$} & \multicolumn{3}{|c|}{ Docentes } & \multirow{2}{*}{$\begin{array}{l}\text { Total de } \\
\text { instituições }\end{array}$} & \multirow{2}{*}{$\begin{array}{l}\text { Mestre/ } \\
\text { instituição }\end{array}$} & \multirow{2}{*}{$\begin{array}{l}\text { Doutor/ } \\
\text { instituição }\end{array}$} \\
\hline & Total & Mestre & Doutor & & & \\
\hline Pública & 12268 & 4378 & 6055 & 21 & 208,5 & 288,3 \\
\hline Federal & 4409 & 1392 & 2484 & 4 & 348,0 & 621,0 \\
\hline Estadual & 7611 & 2886 & 3558 & 13 & 222,0 & 273,7 \\
\hline Municipal & 248 & 100 & 13 & 4 & 25,0 & 3,3 \\
\hline Privada & 16301 & 7464 & 2116 & 164 & 45,5 & 12,9 \\
\hline Paraná (Total) & 28569 & 11842 & 8171 & 185 & 64,0 & 44,2 \\
\hline Sul & 72217 & 30789 & 23090 & 409 & 75,27 & 56,45 \\
\hline
\end{tabular}

Fonte: elaboração própria com base nos dados da Sinopse Estatística da Educação Superior (2011) INEP.

Nota: o mesmo professor pode exercer funções docentes em uma ou mais instituições. 
Na região Sul do Brasil a média de doutores e mestres é de 56,45 contra 273,7 das IES paranaenses, visto que se destaca a alta qualificação do quadro quando comparado a média da região Sul. A média de doutores para as instituições estaduais paranaenses $(222,0)$ também é bastante elevada quando se considera o estado, que tem em média 44,2 doutores por instituição.

As IES estaduais paranaenses (Figura 1) são subdivididas em seis universidades implementadas e uma em fase de implantação, que agrupará sete faculdades estaduais. As três mais antigas universidades estaduais paranaenses são a Universidade Estadual de Londrina (UEL), a Universidade Estadual de Maringá (UEM) e a Universidade Estadual de Ponta Grossa (UEPG), as quais foram criadas no ano de 1969 (UEL, 2013; UEM, 2013; UEPG, 2013). A criação da UEM ocorreu pela integração de três faculdades estaduais que atuavam na cidade de Maringá (UEM, 2013). Atualmente a UEM tem, além de sua sede localizada em Maringá, outros seis campi regionais (Cianorte, Goioerê, Arenito, Diamantina do Norte, Umuarama, Ivaiporã). A UEPG, tal como a UEL, iniciou suas atividades com a junção de cinco faculdades estaduais que atuavam na cidade onde atualmente está instalada (UEL, 2013; UEPG, 2013).

Das universidades implantadas, destaca-se no período recente a Universidade Estadual do Norte do Paraná (UENP), cuja criação aconteceu em 2006 com a reunião de cinco faculdades (UENP, 2013). A sede está localizada na cidade de Jacarezinho com campi em Jacarezinho, Bandeirantes e Cornélio Procópio. Após traçar um panorama das universidades estaduais paranaenses, nota-se a grande heterogeneidade do grupo de docentes que as instituições compõem. 
Figura 1 - Mapa com a localização das universidades estaduais paranaenses (2012)

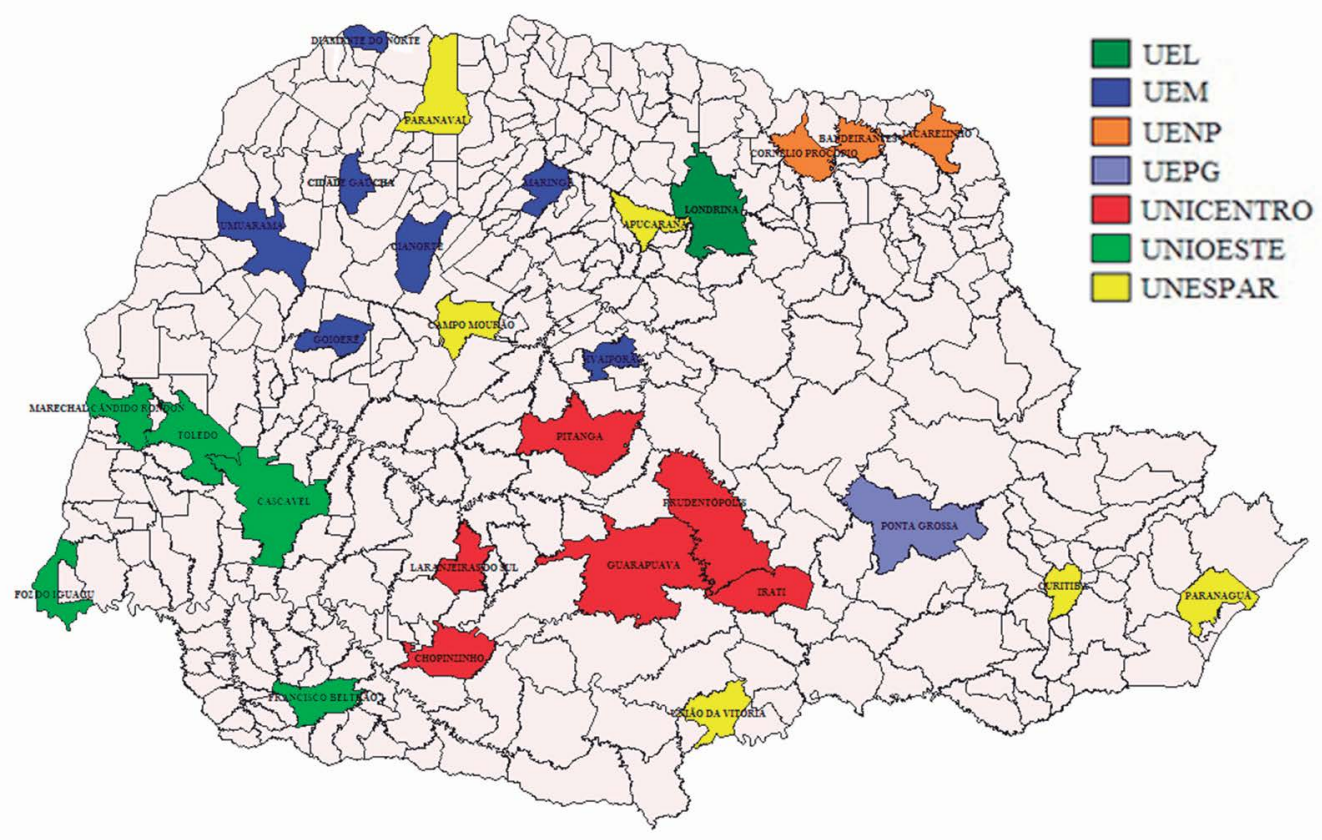

Fonte: elaboração própria com base no Censo Acadêmico do Ensino Superior formulado SETI (2012).

Após traçar um panorama das universidades estaduais paranaenses, nota-se a grande heterogeneidade do grupo de docentes que as instituições compõem. Observando a formação dos docentes em atividade nas universidades estaduais (Gráfico 1), percebe-se que a participação de profissionais sem pós-graduação é muito pequena, atingindo 5\% somente na UENP. A distribuição dos profissionais com especialização e com mestrado, por outra via, mostra-se mais desigual, sendo que nas duas universidades mais novas, UENP e Unespar, as participações são maiores, $19,9 \%$ e $23,5 \%$ de especialistas e $49 \%$ e $55,6 \%$ de mestres, respectivamente. Os docentes com doutorado representam mais da metade dos docentes em atividade na UEL $(59,5 \%)$ e UEM $(60,9 \%)$, todavia, a participação dos doutores é representativa também na UEPG $(46,9 \%)$ e Unioeste $(45,1 \%)$. A instituição com menor participação de doutores é a Unespar, na qual apenas $17,1 \%$ dos docentes possuem o título de doutor.

A alta concentração de doutores na UEL e UEM impacta na quantidade e na qualidade da produção científica, assim como afeta o volume de investimento realizado em pesquisa em tais instituições. 
A despeito das diferenças entre as universidades estaduais paranaenses, no conjunto, os dados do Inep (2011) permitem afirmar que o Paraná detém o segundo maior número de doutores das IES e das universidades estaduais brasileiras, e se comparados ao total de docentes das universidades estaduais na região Sul do país, aproximadamente $86 \%$ dos professores doutores estão lotados nas universidades estaduais no Paraná, o que potencializa a capacidade de produção do conhecimento e seu transbordamento regional.

Gráfico 1 - Formação acadêmica dos docentes por Universidade Estadual do Paraná (2012)

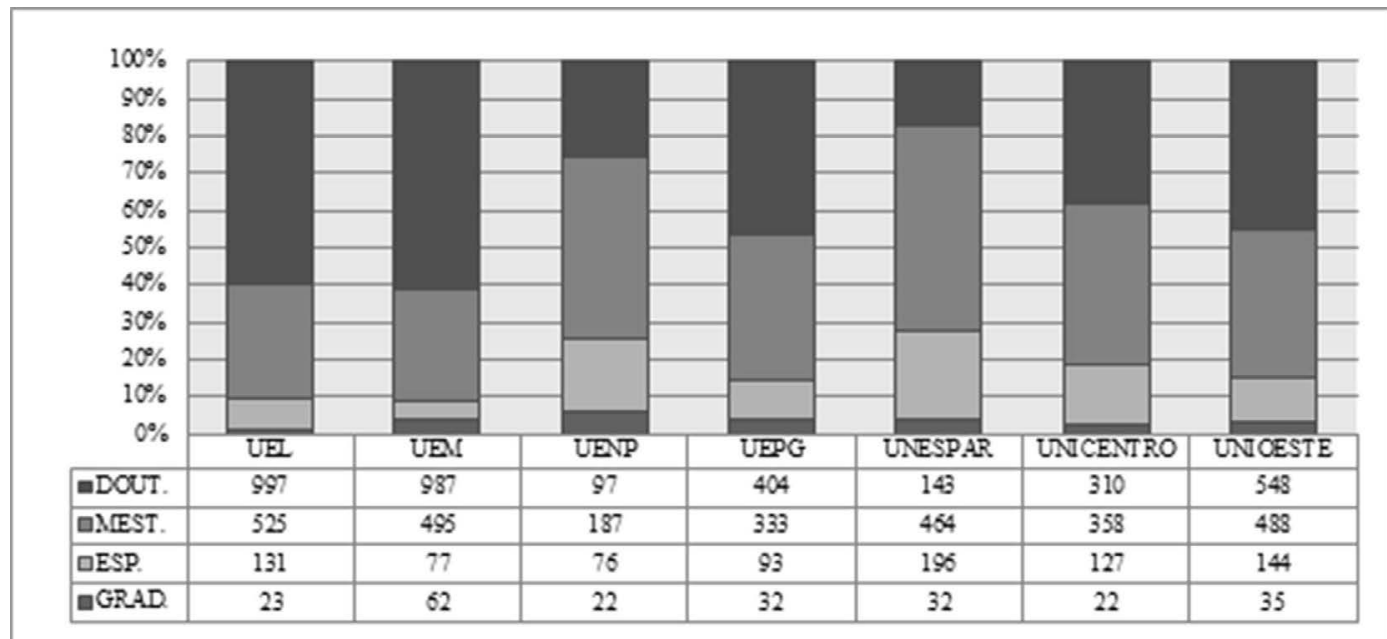

Fonte: elaboração própria com base no Censo Acadêmico do Ensino Superior formulado pelo SETI (2012).

Outra informação importante é o regime de trabalho dos docentes das universidades estaduais paranaenses (Tabela 2), pelo qual se tem o grau de dedicação às atividades acadêmicas. Em 2011, cerca de 89\% dos docentes mantinham tempo integral ao trabalho docente nas IES e universidades estaduais do Paraná (INEP, 2011).

A participação dos docentes com tempo integral e dedicação exclusiva (Tide) é outro fator preponderante na definição da capacidade de produzir conhecimento científico da instituição, isso porque exige do docente a participação em projetos de ensino, pesquisa e extensão, os quais tem por objetivo a produção acadêmica. De acordo com a Tabela 2, a universidade com maior participação de docentes em regime de dedicação exclusiva é a UEM, com $81,5 \%$, seguida pela Unioeste $(75,9 \%)$ e UEL $(72,4 \%)$. A UENP é a instituição com menor proporção de docentes com Tide, sendo que, dos 388 em atividade, apenas 212 estão em regime de dedicação integral. 
Tabela 2 - Participação dos docentes com Tide por Universidade Estadual do Paraná (2013)

\begin{tabular}{l|c|c|c}
\hline \multirow{2}{*}{ IES } & \multicolumn{3}{|c}{ Docentes } \\
\cline { 2 - 4 } & Total & Tide & $\begin{array}{c}\text { Participação dos docentes com } \\
\text { Tide (\%) }\end{array}$ \\
\hline UEL & 1.700 & 1.231 & 72,4 \\
UEM & 1.637 & 1.334 & 81,5 \\
UENP & 388 & 212 & 54,6 \\
UEPG & 894 & 573 & 64,1 \\
Unespar & $\mathrm{n} / \mathrm{d}$ & $\mathrm{n} / \mathrm{d}$ & $\mathrm{n} / \mathrm{d}$ \\
Unicentro & 831 & 502 & 60,4 \\
Unioeste & 1.153 & 875 & 75,9 \\
\hline
\end{tabular}

Fonte: dados obtidos junto às Pró-Reitorias de Recursos Humanos das respectivas Universidades.

Deste modo, a qualificação dos docentes e o tempo dedicado integralmente às instituições paranaenses correspondem às discussões realizadas por Macedo (2005), as quais identificam que a produção científica brasileira tem sua origem nas instituições universitárias e nessas estão concentrada, traço marcante da pesquisa recente brasileira.

No entanto, chama a atenção à participação de cada instituição na oferta de cursos presenciais de graduação. Embora o tamanho das universidades seja irregular, como se observa pelo total de docentes em atividade, a diferença entre a participação da universidade que oferta o maior número de vagas e a que oferta o menor número é de apenas $11 \%$. Em relação ao total de cursos presenciais, a diferença entre a participação da maior e da menor ofertante é de $13 \%$. O resultado da relativa dispersão da oferta de vagas e cursos presenciais de graduação é a discrepância entre vagas e inscritos por processo seletivo. Em 2012, a UEL foi a responsável por disponibilizar o mais elevado número de vagas em cursos presenciais, contudo, a razão entre inscritos e vagas foi a terceira maior. A UEM, a qual ofertou apenas 76 vagas a menos que a UEL, teve uma razão inscritos-vaga quase duas vezes maior, com isso se mostrou a universidade com maior demanda por vagas em cursos de graduação. A UEPG e a Unespar, que têm suas participações na oferta de vagas em cursos de graduação entre as três menores, apresentam a segunda maior razão de inscritos-vaga, ambas com 13,10. 
Gráfico 2 - (a)Porcentagem de vagas em curso de graduação oferecida por IES estadual paranaense - 2012. (b) Porcentagem de cursos presenciais por IES estadual paranaense - 2012.

(a)

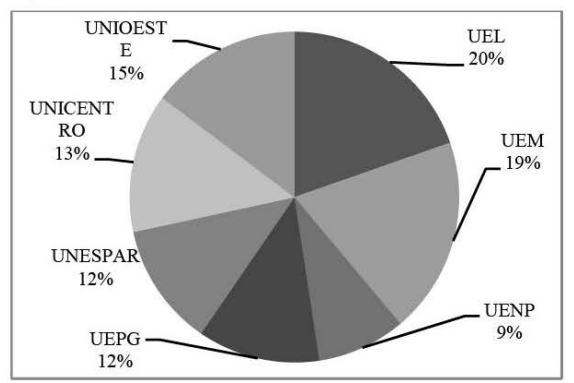

(b)

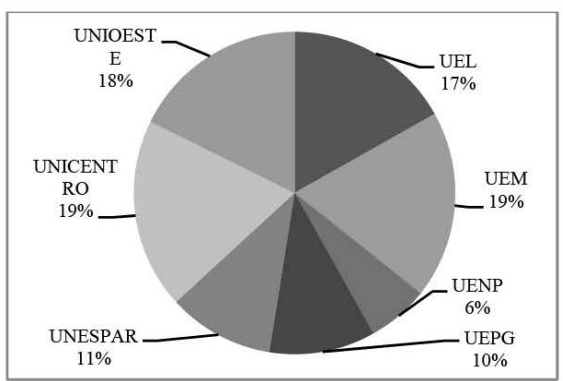

Fonte: elaboração própria com base no Censo Acadêmico do Ensino Superior formulado SETI. 2012. SETI.

De modo geral, a concorrência pelas vagas oferecidas nas universidades estaduais é elevada em todas as instituições. Tal fato pode ser encarado com um quesito importante para a qualidade do ensino e da produção científica das universidades, isso porque permite a seleção dos candidatos mais preparados e produtivos.

Gráfico 3 - Discrepância entre o total de vagas inscritos por IES estadual do Paraná (2012).

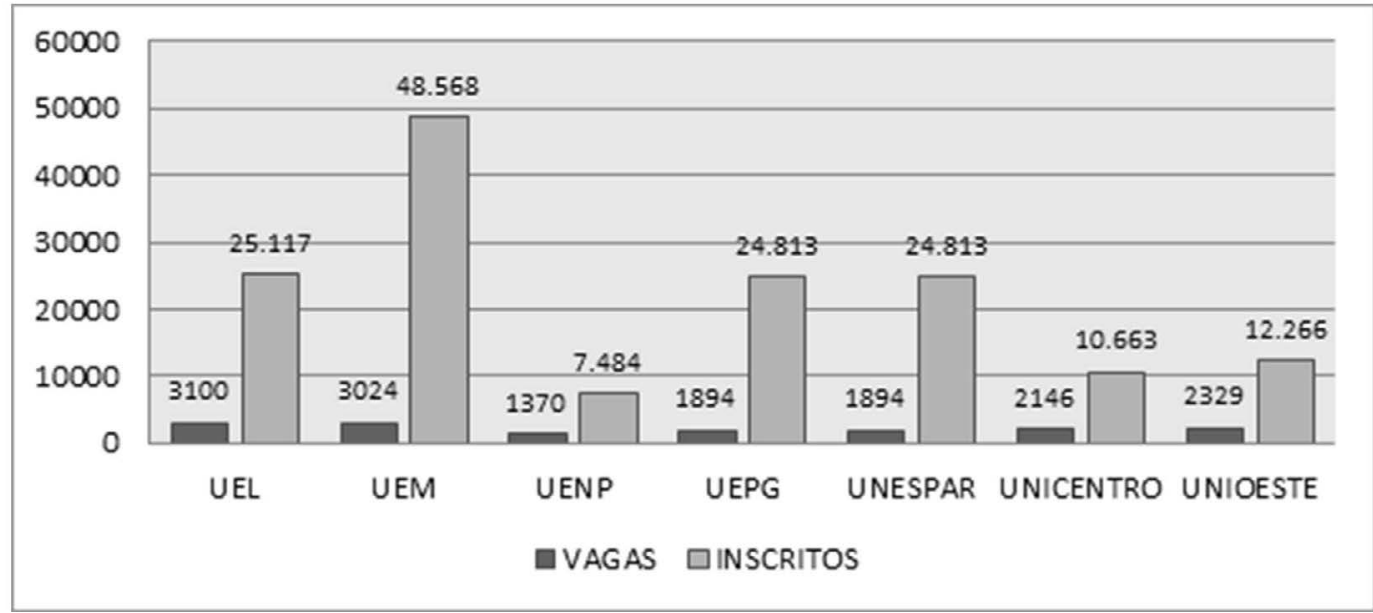

Fonte: elaboração própria com base no Censo Acadêmico do Ensino Superior formulado SETI (2012).

Ao longo desta seção, expostos se expôs um retrato das universidades estaduais paranaenses com atenção especial aos fatores que motivam a produção científica e a sua qualidade. A seguir será analisada a diferença entre as instituições em termos da geração de conhecimento. 


\subsection{Geração de conhecimento nas universidades estaduais do Paraná}

A produção científica de uma universidade está intimamente ligada à quan-

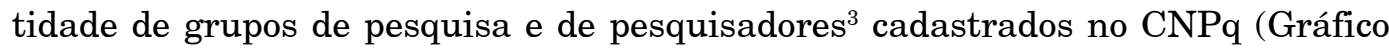
4). Em vista disso, a evolução dessas duas variáveis na década de 2000 é capaz de indicar o desempenho das instituições. Nos anos selecionados, as universidades estaduais paranaenses apresentaram, via de regra, uma excelente atuação, visto que o número de grupos de pesquisa saltou de 347 para 1241, entre 2000 e 2010, o que representa uma taxa de variação média de $25,76 \%$ ao ano. A UEL permaneceu no primeiro lugar em número de grupos praticamente em todo o período, perdendo posição para a UEM somente em 2002.

A elevada quantidade de grupos de pesquisa presente na UEL e na UEM sinaliza uma tradição maior dessas duas instituições na produção científica frente às demais. Todavia, a variação percentual do número de grupos das outras três universidades que estavam implantadas no início da década de 2000 - UEPG, Unicentro e Unioeste - foi consideravelmente superior à das duas primeiras. Além disso, os dados disponíveis para UENP e Unespar também indicam alto crescimento do número de grupos para os anos recentes.

Gráfico 4 - (a) Evolução dos grupos de pesquisa por IES estadual do Paraná de 2000 a 2010. (b) Evolução do número de pesquisadores por IES estadual do Paraná de 2000 a 2010.

(a)

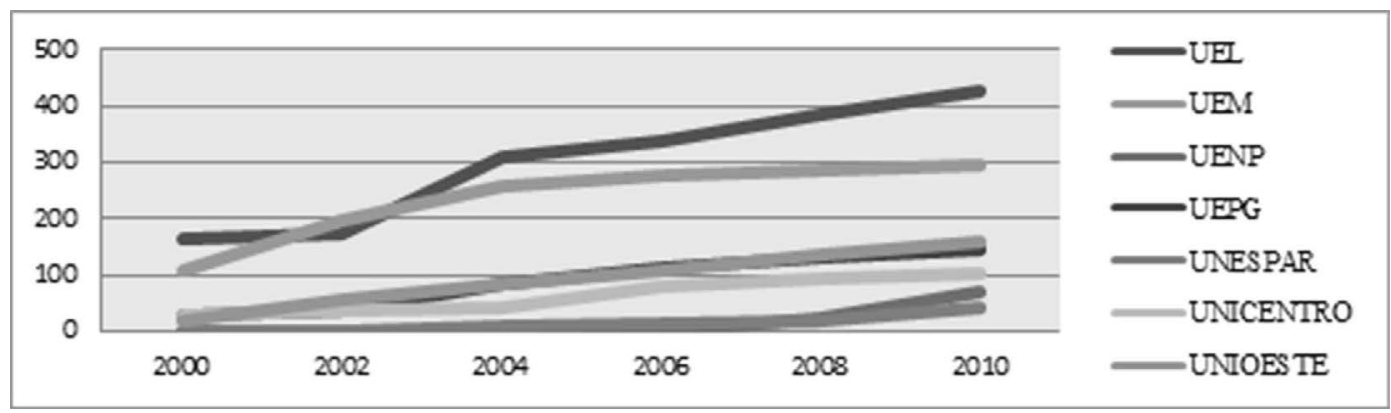


(b)

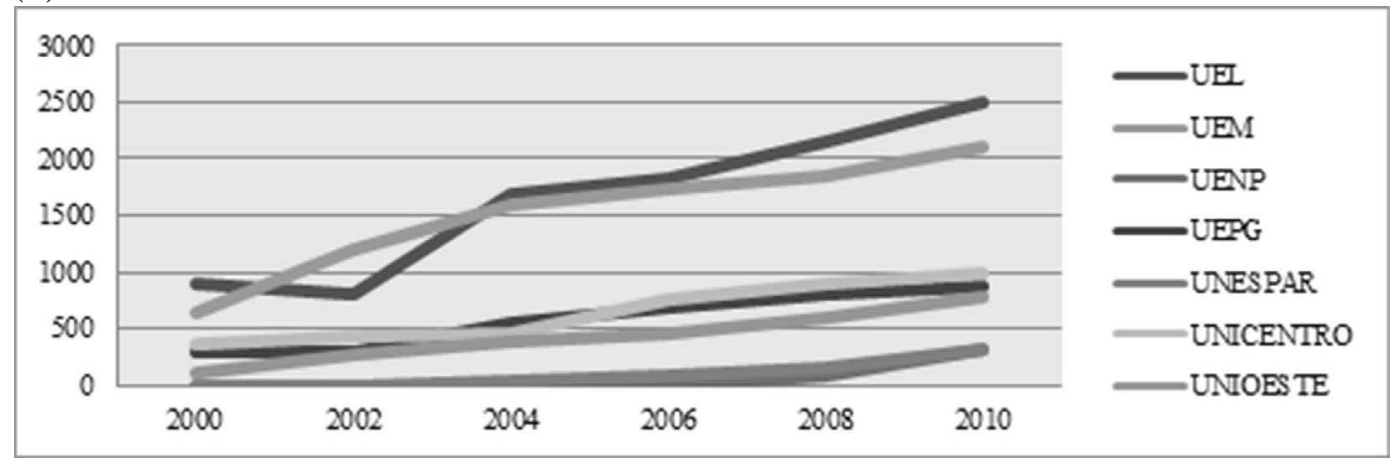

Nota: Em geral, há dupla contagem no número de pesquisadores tendo em vista que o indivíduo que participa de mais de um grupo de pesquisa foi computado mais de uma vez.

Fonte: elaboração própria com base no Plano Tabular do Diretório de Grupos de Pesquisa do CNPq.

O número de pesquisadores por IES basicamente acompanhou a tendência dos grupos de pesquisa. A UEL permaneceu à frente das demais em número de pesquisadores, com exceção de 2002, por toda a década de 2000, no entanto, a Unioeste foi a universidade que apresentou a maior taxa de variação no período, atingindo $574,36 \%$. Quando se toma como referência o período de 2004 a 2010, nota-se, por outro lado, que a taxa de variação do número de pesquisadores da Unespar atingiu $630,23 \%$, sendo esse o melhor desempenho entre as universidades para os seis anos analisados.

A conclusão a que se chega pela análise dos referidos dados é que as sete universidades estaduais do Paraná vêm intensificando a dedicação empregada na produção científica, embora as duas instituições mais tradicionais continuem a reunir o maior número de pesquisadores e grupos de pesquisa.

As linhas de pesquisa identificadas em um IES exprime a diversidade de temas que originam estudos científicos, por consequência, à medida que crescem as linhas de pesquisa de uma universidade, o número de projetos tende a ser ampliado. Observa-se, na Tabela 3, que em 2010 a UEL contava com 1.210 linhas de pesquisa, ficando em primeiro lugar entre as universidades estaduais paranaenses. $\mathrm{O}$ segundo lugar era ocupado pela UEM. Em geral, as duas universidades revezaram a primeira e segunda colocação ao longo de toda a década de 2000 , entretanto, a taxa de variação do número de linhas de pesquisa para essas foi inferior à verificada nas demais. 
Tabela 3 - Taxa de variação das linhas de pesquisa por universidade estadual paranaense e por grande área, em porcentagem (\%): de 2000 a 2010

\begin{tabular}{l|r|r|r|r|r|r|r}
\hline \multicolumn{1}{c|}{ Grande área } & UEL & UEM & UENP & UEPG & Unespar & Unicentro & Unioeste \\
\hline Ciências Agrárias & 128 & 188 & $\mathrm{n} / \mathrm{d}$ & 52 & $\mathrm{n} / \mathrm{d}$ & 360 & 174 \\
Ciências Biológicas & 135 & 99 & $\mathrm{n} / \mathrm{d}$ & 255 & $\mathrm{n} / \mathrm{d}$ & $\mathrm{n} / \mathrm{d}$ & 58 \\
Ciências da Saúde & 181 & 254 & $\mathrm{n} / \mathrm{d}$ & 317 & $\mathrm{n} / \mathrm{d}$ & 2850 & 1900 \\
Ciências Exatas e da Terra & 167 & 236 & $\mathrm{n} / \mathrm{d}$ & 206 & $\mathrm{n} / \mathrm{d}$ & 482 & $\mathrm{n} / \mathrm{d}$ \\
Ciências Humanas & 304 & 324 & $\mathrm{n} / \mathrm{d}$ & 279 & $\mathrm{n} / \mathrm{d}$ & 314 & 1420 \\
Ciências Sociais Aplicadas & 306 & 267 & $\mathrm{n} / \mathrm{d}$ & 325 & $\mathrm{n} / \mathrm{d}$ & 172 & $\mathrm{n} / \mathrm{d}$ \\
Engenharias & 129 & 273 & $\mathrm{n} / \mathrm{d}$ & 247 & $\mathrm{n} / \mathrm{d}$ & 1000 & $\mathrm{n} / \mathrm{d}$ \\
Linguística, Letras e Artes & 264 & $\mathrm{n} / \mathrm{d}$ & $\mathrm{n} / \mathrm{d}$ & $\mathrm{n} / \mathrm{d}$ & $\mathrm{n} / \mathrm{d}$ & 182 & 5 \\
TOTAL & 185 & 223 & $\mathrm{n} / \mathrm{d}$ & 227 & $\mathrm{n} / \mathrm{d}$ & 397 & 259 \\
\hline
\end{tabular}

Fonte: elaboração própria com base no Plano Tabular do Diretório de Grupos de Pesquisa do CNPq.

Ao se averiguar o comportamento das linhas de pesquisa por grande área do conhecimento fica evidente que a expansão do número total de linhas foi acompanhada pela diversificação das áreas. Áreas poucos tradicionais nas instituições paranaenses apresentaram taxas de variação consideráveis, tal como é o caso das Ciências Sociais Aplicadas na UEL e UEPG; Engenharias na UEM, UEPG e Unicentro; Ciências Exatas e da Terra na Unicentro; e UEM e Ciências da Saúde na UEPG, Unicentro e Unioeste.

A participação dos investimentos na UEL e na UEM no total de investimento realizados pelo CNPq nas universidades estaduais paranaenses (Gráfico 5), entre 2006 e 2012, reflete a tradição dessas na pesquisa e na produção de conhecimento. Em 2006, as duas universidades recebiam mais de $87,4 \%$ do investimento e $77,41 \%$ em 2010, mesmo com a participação crescente das demais. É interessante ressaltar que a UEM recebeu aproximadamente $50 \%$ de todo o investimento realizado nas universidades estaduais em cada um dos seis anos considerados, apesar da UEL apresentar maior número de pesquisadores e grupos de pesquisa. 
Gráfico 5 - Participação dos investimentos realizados pelo CNPq em bolsas no país e no exterior e fomento à pesquisa nas universidades estaduais paranaenses de 2006 a2012

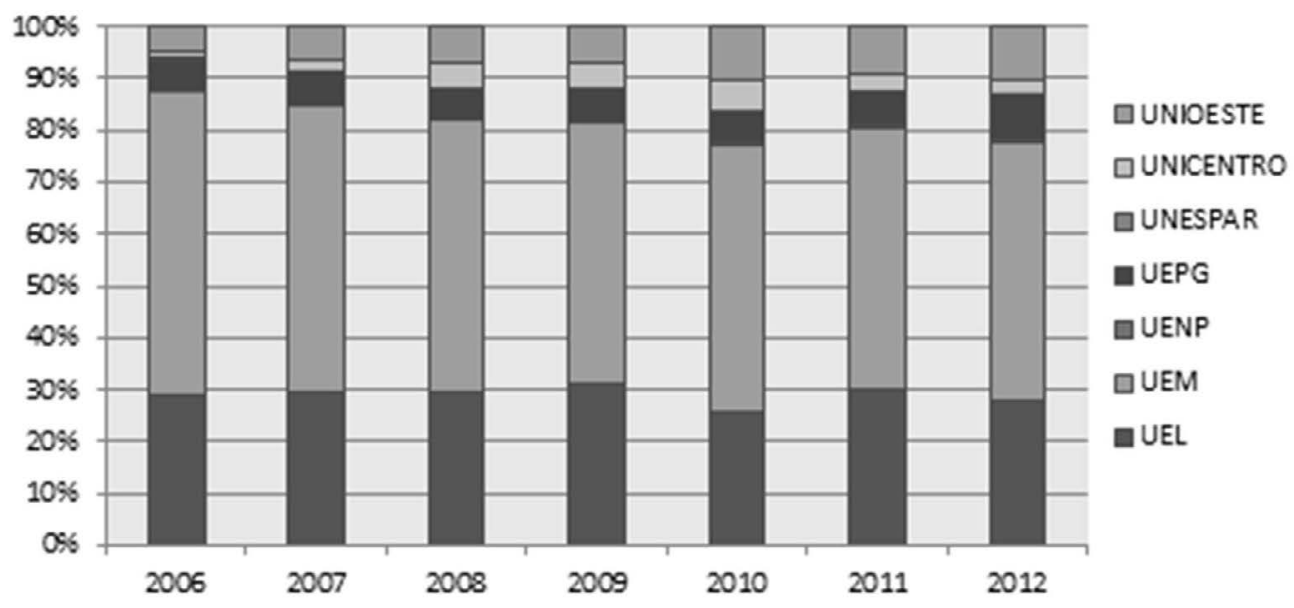

Fonte: elaboração própria com base no CNPq/AEI.

Nota: Inclui recursos dos fundos setoriais; as bolsas de curta duração foram consideradas no Fomento à Pesquisa.

Em conformidade com os dados do Gráfico 5, a porcentagem de bolsas-ano da UEM manteve-se entre $60 \%$ e 50\%, entre 2006 e 2012, enquanto a UEL passava de $30,6 \%$ para $27,11 \%$. Os dados do Gráfico 6 revelam, ainda, o que pode ser uma tendência de aproximação das instituições, como se pode observar pela queda na participação das bolsas-ano recebidas pela UEL e UEM nos últimos anos, enquanto a participação das demais universidades vêm crescendo consideravelmente.

Gráfico 6 - Porcentagem de bolsas-ano no país e no exterior da UEL, UEM e demais universidades estaduais do Paraná de 2006 a 2012

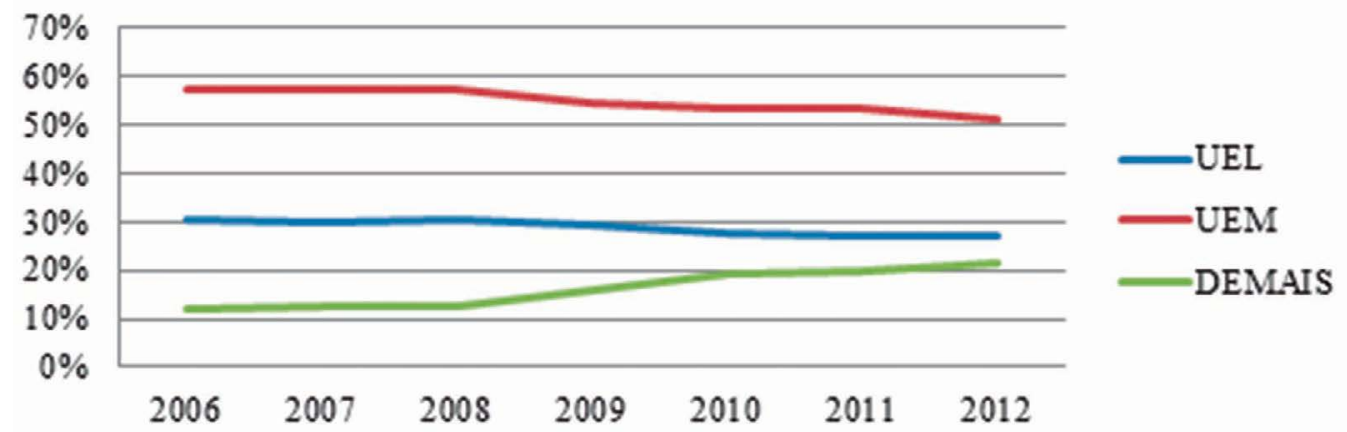

Nota : Inclui recursos dos fundos setoriais; o $\mathrm{n}^{\circ}$ de bolsas-ano representa a média aritmética do $\mathrm{n}^{\circ}$ de mensalidades pagas de janeiro a dezembro.

Fonte: elaboração própria com base no CNPq/AEl. 
Colocando em foco os programas de pós-graduação, constata-se, mais uma vez, a tradição das universidades de Londrina e Maringá, como nota-se pelo elevado número de bolsas concedidas pela Coordenação de Aperfeiçoamento de Pessoal de Nível Superior (Capes). Das 2.632 bolsas da Capes repassadas para as universidades estaduais paranaenses, tem-se que 38,3\% destinavam-se à UEM e 32,6\% à UEL. Isso significa que, sozinhas, as duas instituições receberam 70,9\% das bolsas. A UENP apenas 0,42\% das bolsas concedidas pelas Capes em 2012.

Tabela 4 - Concessão de bolsas de pós-graduação feita pela Capes para as universidades estaduais paranaenses (2012)

\begin{tabular}{|c|c|}
\hline Universidade & n. de bolsas \\
\hline UEL & 859 \\
\hline UEM & 1008 \\
\hline UENP & 11 \\
\hline UEPG & 278 \\
\hline Unicentro & 130 \\
\hline Unioeste & 346 \\
\hline
\end{tabular}

Fonte: elaboração própria com base nos dados do GeoCapes. 2012. Capes.

A assimetria na concessão de bolsas não se deve somente à quantidade de programas de pós-graduação presentes em cada universidade, como exposto na Tabela 5, uma vez que a primeira instituição em quantidade de programas, a UEL, foi a segunda em número de bolsas. Isso se deve ao fato das bolsas estarem diretamente ligadas à avaliação realizada pela Capes.

As bolsas são concedidas às instituições com programas de pós-graduação stricto sensu que obtiveram nota igual ou superior a três na avaliação (CAPES, 2013), considerando-se, entre outros itens, a produção técnica, a produção e formação dos docentes, as linhas de pesquisa, a produção bibliográfica e os projetos de pesquisa (CAPES, 2013). As notas são distribuídas em uma escala de 1 a 7 , sendo as notas 6 e 7 conferidas aos programas com nível de excelência equivalente ao dosmais importantes centros internacionais. A nota 5 indica alto nível de desempenho e é o maior conceito cedido a programas que ofereçam apenas mestrado, ademais, as notas 4 e 3 sinalizam, respectivamente, bom desempenho e desempenho regular. Por fim, para os programas que são designados as notas 1 e 2 não se concede a renovação de reconhecimento de seus cursos, uma vez que o desempenho é entendido como abaixo do padrão de qualidade requerido (CAPES, 2013).

Entre as universidades estaduais do Paraná, em 2012, o conceito máximo obtido era 6, o qual foi concedido a dois programas de mestrado/doutorado, inscritos na UEM, e, portanto, a UEM apresenta-se como a única universidade estadual 
paranaense com programas de pós-graduação com excelência internacional. A nota 5, por sua vez, foi outorgada a 11 programas distribuídos entre a UEL e a UEM. A UEPG e a Unioeste receberam notas 4 e 3, enquanto a UENP e a Unicentro, as duas instituições mais novas da lista, receberam apenas conceito 3 .

Tabela 5 - Número de programas de pós-graduação por universidade estadual paranaense e por nota da Capes (2012)

\begin{tabular}{l|r|r|c|c|c|c}
\hline \multicolumn{1}{c|}{ Nota } & UEL & UEM & UENP & UEPG & Unicentro & Unioeste \\
\hline 3 & 20 & 11 & 2 & 7 & 8 & 16 \\
4 & 13 & 13 & - & 6 & - & 4 \\
5 & 6 & 5 & - & - & - & - \\
6 & - & 2 & - & - & - & - \\
Total & 39 & 31 & 2 & 13 & 8 & 20 \\
\hline
\end{tabular}

Fonte: elaboração própria com base nos dados do GeoCapes (2012). Capes..

Como mencionado acima, um dos quesitos avaliados pela Capes é a produção bibliográfica dos programas dos programas de pós-graduação. A Tabela 6 traz a evolução dos artigos publicados em periódicos em cada universidade considerando trabalhos com autoria de professores e estudantes tanto de graduação quanto de pós-graduação.

Tabela 6 - Artigos completos publicados segundo circulação do periódico por universidade estadual do Paraná, de 2000 a 2010 ou (2000 -2010)

\begin{tabular}{l|c|c|c|c|c|c}
\hline \multirow{2}{*}{ IES } & \multicolumn{2}{|c|}{$2000-2003$} & \multicolumn{2}{c|}{$2003-2006$} & \multicolumn{2}{c}{$2007-2010$} \\
\cline { 2 - 7 } & $\begin{array}{c}\text { Circulação } \\
\text { Nacional }\end{array}$ & $\begin{array}{c}\text { Circulação } \\
\text { Internacional }\end{array}$ & $\begin{array}{c}\text { Circulação } \\
\text { Nacional }\end{array}$ & $\begin{array}{c}\text { Circulação } \\
\text { Internacional }\end{array}$ & $\begin{array}{c}\text { Circulação } \\
\text { Nacional }\end{array}$ & $\begin{array}{c}\text { Circulação } \\
\text { Internacional }\end{array}$ \\
\hline UEL & 3.903 & 1.398 & 4.548 & 2.790 & 6.587 & 4.713 \\
UEM & 4.595 & 1.811 & 5.340 & 3.153 & 6.621 & 4.782 \\
UENP & $\mathrm{n} / \mathrm{d}$ & $\mathrm{n} / \mathrm{d}$ & $\mathrm{n} / \mathrm{d}$ & $\mathrm{n} / \mathrm{d}$ & 489 & 76 \\
UEPG & 1.061 & 356 & 1.760 & 776 & 2.186 & 1.691 \\
Unespar & 77 & 2 & 233 & 23 & 315 & 95 \\
Unicentro & 589 & 201 & 1.018 & 416 & 2.139 & 591 \\
Unioeste & 1.547 & 183 & 2.379 & 299 & 3.686 & 880 \\
\hline
\end{tabular}

Nota: Produção bibliográfica refere-se ao somatório das produções de pesquisadores e estudantes.

Fonte: elaboração própria com base no Plano Tabular do Diretor de Grupos de Pesquisa do CNPq.

No período compreendido entre 2000 e 2010, as universidades UEL e UEM mantiveram-se amplamente a frente das outras cinco universidades nesse quesito. A UEM esteve na liderança ao longo dos dez anos considerados, sendo que a diferença entre essa e a UEL cresceu entre 2003 e 2006 para reduzir-se bruscamente de 2007 a 2010. A participação das publicações em periódicos de circulação nacional 
da UEL e da UEM alcançou 72,19\% do total das publicações das universidades estaduais paranaenses entre 2000 e 2003, queda de 64,72\%, com o que encerrou o período em $59,98 \%$. A participação das publicações em periódicos internacionais seguiu 'a mesma tendência de queda no decorrer da década, sinalizando a crescente dedicação das demais universidades à produção científica.

\section{Conclusão}

Os resultados apontam as universidades estaduais paranaenses como principais atores na geração de produção científica, oferta de ensino superior e que concentra a maioria dos recursos humanos de alta qualificação no estado, em termos de ensino e pesquisa acadêmica. Também pode-se concluir com este estudo que há assimetrias regionais significativas entre as instituições estudadas, tanto na oferta de vagas quanto na produção científica a partir dos indicadores analisados, com destaque para os indicadores da UEL e UEM e Unioeste.

Do ponto de vista da sua presença no contexto brasileiro e da região sul, tanto as IES em seu conjunto, quanto as universidades estaduais paranaenses têm significativa representatividade. São essas universidades que, sob características específicas do Brasil, realizam preponderantemente a pesquisa científica.

O desafio que este estudo destaca é a importância do ensino e pesquisa como formas de acumulo de capital humano ou aporte para tal. Assim, traçado o perfil da produção científica e ensino superior no Paraná pode-se destacar a importância das IES como indutoras neste processo. Este estudo descortina que a estrutura para tal existe e que as IES no estado podem capitanear esse processo.

Apesar disso, este trabalho chama a atenção para o fato de que as universidades estejam atentas aos modos de produção do conhecimento e aos mecanismos de interação com o sistema produtivo. Ao assim fazer tornam-se importantes parceiras do desenvolvimento regional, particularmente pela identificação de estudos e projetos que tenham significado para a região em que estão inseridas.

Como uma dificuldade a ser superada, destaca-se a relativa assimetria entre as instituições, com o que, pode-se observar que as mais antigas absorvem mais recursos e apresentam indicadores relacionados à geração do conhecimento mais positivos. Assim, aponta-se a necessidade de uma maior interação entre essas instituições no sentido de superar tal desafio. 


\title{
Analysis of the scientific production and knowledge generation for the Paraná states universities
}

\begin{abstract}
The objective of this study is to analyze the contribution of the state universities for scientific production and allocation of resources in the state of Paraná. We used several data sources, including data from the institutions themselves; database CNPq; Capes and the Higher Education Census, conducted by the Department of Science and Technology of Paraná (SETI). The results indicate that Paraná state universities as key actors in the generation of scientific production and supply of higher education that concentrates most highly qualified human resources in the state in terms of education and academic research. Also it can be concluded from this study that there are differences between the institutions studied, both in the number of vacancies as scientific production from the analyzed indicators, especially indicators of UEL, UEM and Unioeste.
\end{abstract}

Keywords: State Universities. Scientific production. Higher education.

\section{Análisis de indicadores de producción científica y generation de conocimiento en las universidades estatales de Paraná}

\section{Resumén}

El objetivo de este estudio es analizar la contribución de las universidades estatales para la producción científica y la oferta de educación superior en el estado de Paraná. Se utilizaran diversos bancos de datos, entre ellos, datos de las instituciones estudiadas; datos del banco de CNPq; Capes y del Censo de Educación Superior, realizado pela secretaria de ciencias y tecnología de Paraná (SETI). Los resultados señalan a las universidades estatales paranaenses como las principales impulsoras en la elaboración de producción científica, oferta de educación superior y concentra la mayoría de recursos humanos de alta calificación dentro del estado, en términos de enseñanza y pesquisa académica. También, se puede concluir con este estudio que hay muchas diferencias entre las universidades estudiadas, principalmente en las vacantes ofrecidas y en la producción científica a partir de los indicadores analizados, con destaque principalmente para los indicadores de la UEL, UEM y Unioeste.

Palabras chave: Universidades. Producción científica. Educación superior. 


\section{Notas}

1 Ver teoria da sinalização e externalidades associadas à educação e à acumulação de capital humano em Barbosa Filho e Pessoa (2010).

2 Para mais informações consultar o Plano Tabular do Diretório de Grupos de Pesquisa no Brasil do CNPq para os anos de 2000 a 2010.

3 De acordo com a definição dada pelo CNPq, a qual pode ser encontrada no Diretório dos Grupos de Pesquisa no Brasil, pesquisadores são profissionais graduados ou pós-graduados membros de uma equipe de pesquisa. Caso esteja matriculado em um curso de graduação ou de pós-graduação, o indivíduo é identificado como estudante

\section{Referências}

ACEMOGLU, D.; JOHNSON, S. H.; ROBINSON, J. A. Unbundling Institutions. Journal of Political Economy, Chicago, v. 13, n. 5, p. 949-995, out. 2005.

ACS, Z. J.; ANSELIN, L.; VARGA, A. Patents and Innovation Counts as Measures of Regional Production of New Knowledge. Research Policy. v. 31, n. 3, p. 1069-1085, 2002.

ACS, Z. J.; AUDRETSCH, D. B.; FELDMAN, M. P. R\&D Spillovers and Recipient Firm Size. Review of Economics and Statistics, Cambridge, v. 76, n. 4, p. 336-340, maio 1994.

ALVAREZ, R. B.; KANNEBLEY JÚNIOR, Sérgio; CAROLO, M. D. O impacto da interação universidade-empresa na produtividade dos pesquisadores: uma análise para as ciências exatas e da terra nas universidades estaduais paulistas. Revista Brasileira de Inovação, Campinas-SP, v. 12, n. 1, p. 171-206, jan./jun. 2013.

ANSELIN, L.; VARGA, A.; ACS, Z.J. Geographical spillovers and university research: a spatial econometric perspective. Growth and Change, Kentucky, v. 31, n. 4, p. 501-515, 2000.

BARBOSA FILHO, F.; PESSOA, S. Educação e Crescimento: O que a Evidência Empírica e Teórica Mostra? Economia, Brasília, v. 11, n. 2, p. 265-303, maio/ago. 2010.

BECKER, G. Investment in human capital: a theoretical analysis. Journal of Political Economy, Chicago, v. 70, n. 5, p. 9-49, out. 1962.

CAPES. Cadernos de indicadores. Disponível em: <http://conteudoweb.capes.gov.br/ conteudoweb/CadernoAvaliacaoServlet>. Acesso em: 23 ago. 2013.

CAPES. Resultado da avaliação de programas: relatório final. Disponível em: <http://www.capes.gov.br/avaliacao/resultados-da-avaliacao-de-programas/2315>. Acesso em: 23 ago. 2013.

CHIARINI, T.; VIEIRA, K. P.; ZORZIN, P. G. Universidades Federais Mineiras: análise da produção da pesquisa científica e conhecimento no contexto do sistema mineiro de inovação. Nova Economia, Belo Horizonte, v. 22, n. 2, p. 307-332, maio/ago. 2012.

FLORAX, R. The University: A Regional Booster? Economic Impacts of Academic Knowledge Infrastructure. Avebury: Aldershot, 1992.

FLORAX, R. R. ; FOLMER, H. Knowledge impacts of universities on industries: An aggregate simultaneous investment model. Journal of Regional Science, local de publicação, v. 32 , p. 437- 466, 1992. 
GODDARD, J. B. Universities and regional development: an overview. In: GRAY (Ed) Universities and the Creation of Wealth. Milton Keynes, Open University Press. 1999.

GODDARD, J. B.; ASHEIN, B.T.; CRONBERG, T.; VIRTANEN, I. Learning regional engagement: a Re-evaluation of the Third Role of Eastern Finland University. Finnish Higher Education Evaluation Council (FINHEEC), Helsinki, 2003.

IPARDES - INSTITUTO PARANAENSE DE DESENVOLVIMENTO ECONÔMICO E SOCIAL. Municípios e regiões. Disponível em: <http://www.ipardes.gov.br >. Acesso em: 14 jan. 2014.

KROTH, D. C.; DIAS, J. Os efeitos dos investimentos público e privado em capitais físico e humano sobre o produto per capita dos municípios da região Sul: uma análise em painéis de dados dinâmicos, 1999-2004. Nova Economia, Belo Horizonte, v. 22, n. 3, p. 821-849, set./ dez. 2012.

LOPES, Roberto Paulo M. Universidade e economias de aglomeração: as dimensões econômicas e espaciais geradas pelo consumo de ensino superior em Vitória da Conquista. In: ENCONTRO DE ECONOMIA BAIANA, VI, 2010. Salvador. Anais... Salvador: UFBA/ Desenbahia, 2010.

LOPES, Roberto Paulo M. Universidade pública e desenvolvimento local: uma abordagem a partir dos gastos da Universidade Estadual do Sudoeste da Bahia. Vitória da Conquista: Edições Uesb, 2003.

MACEDO, A. R. O papel social da universidade. Revista da ABMES, Brasília, v. 23 , n. 34 , p. 7-12, abr. 2005.

MINCER, J. Investment in human capital and personal income distribution. Journal of Political Economy, Chicago, v. 66, n. 4, p. 281-302, ago. 1958.

MINISTÉRIO DO TRABALHO E EMPREGO - MTE. Cadastro Geral de Empregados e Desempregados - CAGED. Disponível em:<http://portal.mte.gov.br/caged/ >. Acesso em: 10 jan. 2014.

NAKABASHI, L.; FELIPE, E. Capital humano nos municípios paranaenses. Revista Análise Econômica (UFRGS), Porto Alegre, v. 25, n. 47, p. 7-22, set. 2007.

NELSON, R.; PHELPS, E. Investment in humans, technological diffusion and economic growth. American Economic Review: papers and proceedings, 61:69-75, 1966.

OCDE. Relatório da participação do estado do Paraná no projeto OCDE: apoio das instituições de ensino superior ao desenvolvimento regional. Curitiba, ago. 2007.

RAPINI, Márcia Siqueira. Interação universidade-empresa no Brasil: evidências do Diretório dos Grupos de Pesquisa do CNPq. Estudos Econômicos, São Paulo, v. 37, n. 1, p. 211-233, jan./mar. 2007.

REITORIA da Unespar será instalada em Paranavaí, decidem deputados. G1, [S.I.], 4 de jun. 2012. Disponível em: <http://g1.globo.com/pr/parana/noticia/2013/06/reitoria-da-unespar-sera-instalada-em-paranavai-decidem-deputados.html>. Acesso em: 24 de ago. 2013.

ROMER, D. Advanced macroeconomics. 2. ed., Nova York: McGraw-Hill. 2001.

. Endogenous technological change. The Journal of Political Economy, Chicago, v. 98 n. 5, p. 71-102, dez. 1989. 
SCHULTZ, T. W. Capital formation by education. The Journal of Political Economy, Chicago, v. 68, n. 6, p. 571-583, dez. 1960.

SECRETARIA DA CIÊNCIA E TECNOLOGIA E ENSINO SUPERIOR DO PARANÁ SETI. Instituições de Ensino Superior. Disponível em:<http://www.seti.pr.gov.br/>. Acesso em: 15 jan. 2014.

SILVA, W. P. Os efeitos da educação sobre o diferencial de salários dos mercados de trabalho formal e informal, no Estado do Paraná, em 2009. In: Encontro Nacional ENABER, XI., 2013, Foz do Iguaçu. Anais eletrônicos... Foz do Iguaçu: Questões espaciais no mercado de trabalho, 2013. Disponível em:<http://iclips.no-ip.biz:8001/enaber/lista_artigos.aspx>. Acesso em: 23 jun. 2014.

SILVA, D de G. ; MCCOMB, R. P. Research Universities And Regional High-Tech Firm Start-Up And Exit. Economic Inquiry, Western Economic Association International, Foutain Valley, v. 50, n.1, p. 112-130, jan. 2012.

SOARES, M. S. A. (Coord). A educação superior no Brasil. Porto Alegre: IESALC UNESCO, Instituto Internacional para a Educação Superior na América Latina e no Caribe, 2002.

SOUZA, J. Educação e qualificação profissional como determinantes de inserção no mercado de trabalho brasileiro. In: ENCONTRO REGIONAL DE ECONOMIA ANPEC SUL, XI, 2008, Curitiba. Anais eletrônicos... Curitiba: Econometria, Economia Matemática e Modelagem Quantitativa, 2008. Disponível em:<http://www.economiaetecnologia.ufpr.br/ XI_ANPEC-Sul/artigos_aceitos.html>. Acesso em: 15 jun. 2014.

UEL. História. Disponível em: <http://www.uel.br/gabinete/?content=historico.html>. Acesso em: 24 ago. 2013.

Tempo Integral de Dedicação Exclusiva. Disponível em: <http://www.uel.br/prorh/ ?content=carreira/tidedoc.html>. Acesso em: 24 ago. 2013.

UEM. Histórico. Disponível em: <http://www.uem.br/index.php?option= com_content\&task =view\&id=55\&Itemid=205>. Acesso em: 19 ago. 2013.

UENP. A UENP. Disponível em: <http://www.uenp.edu.br/index.php/institucional>. Acesso em: 20 ago. 2013.

UEPG. Histórico. Disponível em: <http://portal.uepg.br/institucional/universidade/ historico.php>. Acesso em: 20 ago. 2013.

UNICENTRO. História da Unicentro. Disponível em: <http://www2.unicentro.br/historia/>. Acesso em: 20 ago. 2013.

VILLELA, TAIS N.; MAGACHO, LYGIA A. M. Abordagem histórica do sistema de inovação e o papel das incubadoras de empresas na interação dos agentes deste sistema. In: SEMINÁRIO NACIONAL DE PARQUES TECNOLÓGICOS E INCUBADORAS DE EMPRESAS, XIX, 2009. Anais... Florianóplis. Disponível em: http://www.redetec.org.br/publique/ media/PUC-Rio-T-2_1.pdf.

ZAIST, J. K. I. V.; NAKABASHI, L.; SALVATO, M. A. Retornos privados da escolaridade no Paraná. Economia, Brasília, v. 11, n. 1, p.175-198, jan./abr. 2010. 